\title{
EIGHT PARAMETRIC RESONANCES IN A MULTI-FREQUENCY WIDE- BAND MEMS PIEZOELECTRIC VIBRATION ENERGY HARVESTER
}

\author{
Yu Jia ${ }^{l, 2}$, Sijun Du ${ }^{l}$, and Ashwin A Seshia ${ }^{l}$ \\ ${ }^{1}$ Nanoscience Centre, University of Cambridge, Cambridge, UK \\ ${ }^{2}$ Department of Mechanical Engineering, University of Chester, Chester, UK
}

\begin{abstract}
This paper presents a multi-order parametric resonant MEMS piezoelectric disk membrane, for the purpose of broadening the operational frequency bandwidth of a vibration energy harvester by employing the nonlinearityinduced bandwidth broadening associated with this phenomenon as well as the multi-frequency response associated with the higher orders. The fundamental mode $-3 \mathrm{~dB}$ bandwidth at $2.0 \mathrm{~g}$ recorded $55 \mathrm{~Hz}$, while the first parametric resonant peak exhibited $365 \mathrm{~Hz}$ and the $-3 \mathrm{~dB}$ of the first 8 orders accumulated to $604 \mathrm{~Hz}$. The membrane parametric resonator also experimentally demonstrated over 3 -folds improvement in power density compared to a conventional direct resonator (cantilever), when subjected to band-limited white noise.
\end{abstract}

\section{INTRODUCTION}

The conventional approach within the field of vibration energy harvesting employs a directly excited resonator, which typically has a confined operational frequency bandwidth [1]. A compromise between peak power and frequency bandwidth is usually made through adjusting the quality factor for a given resonator. On the other hand, the use of parametric resonance in MEMS electrostatic harvesters has been previously shown [2] to enable multi-frequency operation, while demonstrating both higher power amplitude and wider frequency bandwidth than its directly excited counterpart. Furthermore, with lower damping (under vacuum conditions), the peak power and the frequency bandwidth simultaneously increases for a parametric resonator.

Parametric resonance [3] is a nonlinear vibratory phenomenon associated with periodic modulation in a system parameter resulting in unstable growth of oscillations ultimately bounded by nonlinear effects. Governed by the Mathieu equation (equation 1), under the right conditions, internal amplification of the resulting response to external excitation enables accumulation of significantly more energy within the device than possible through directly excited resonance [4].

$$
\ddot{x}+c \dot{x}+(\delta+2 \epsilon \cos 2 t) x=0
$$

Where, $x$ is the displacement, $c$ is the damping coefficient, $\delta$ relates to natural frequency squared, $\epsilon$ relates to the amplitude of parametric excitation and $t$ is the time domain. Various orders of parametric resonances are theoretically predicted when the excitation frequency is in the vicinity of $2 \omega_{0} / n$, where $\omega_{0}$ is the natural frequency and $n$ is a positive integer denoting the order number. Figure 1 illustrates the stability chart (Strutt diagram) of the Mathieu equation for the first eight orders of parametric resonance. It can be noted that higher orders are less accessible due to the narrowing frequency bands

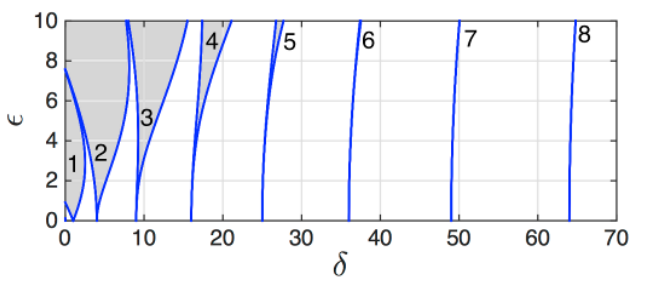

Figure 1: Strutt diagram of the first 8 orders of an undamped Mathieu oscillator. Shaded areas represent regions where conditions are favourable to yield the onset of parametric resonance.

The incorporation of this nonlinear vibration principle at the MEMS-scale has the potential to access multiple higher orders [5] at regular frequency intervals. This study further demonstrates the potential of utilizing multiple orders of parametric resonance as a means of notably enhancing the intrinsic operational frequency bandwidth for a piezoelectric vibration energy harvester.

\section{METHOD}

A circular disk membrane design topology (device shown in figure 2) is adopted based on inspiration drawn from membrane-based musical instruments, such as timpani, which has a high number of resonant modes [6].

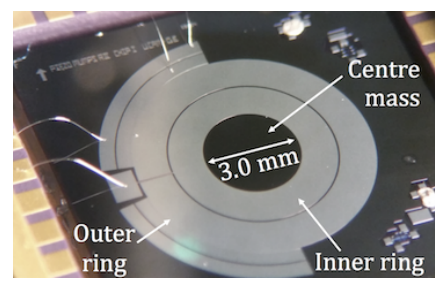

Figure 2: Photograph of the disk membrane MEMS piezoelectric vibration energy harvester capable of being driven into parametric resonance.

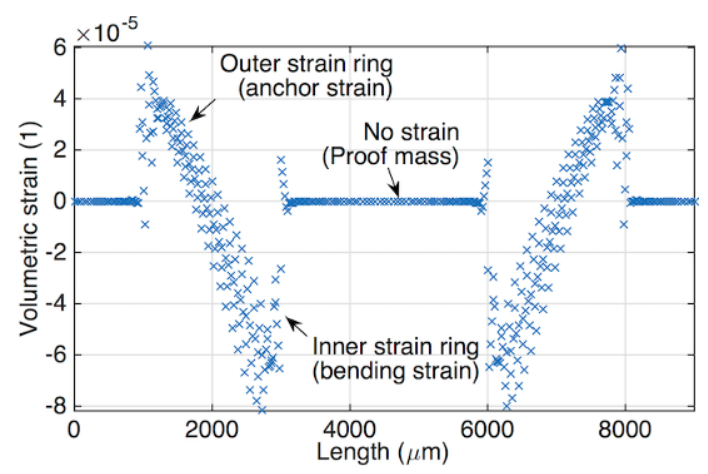

Figure 3: COMSOL simulated strain distribution along the radial length, showing opposing strain rings between the anchor (sides) and the centred proof mass. 
Due to opposing strain polarity of the inner ring (bending strain) and outer ring (anchor strain) of the disk membrane during oscillation, separate active piezoelectric regions were established through electrode segmentation. The boundary of this segmentation was determined through COMSOL simulation of the design (figure 3).

Figure 4 outlines the material stack of the piezoelectric device, comprising of a $0.5 \mu \mathrm{m}$ AlN layer (piezoelectric transduction layer) on a $10 \mu \mathrm{m}$ doped $\mathrm{Si}$ layer (active mechanical device layer/bottom common electrode). Certain regions of the un-etched silicon handle wafer was utilised as the centred proof mass.

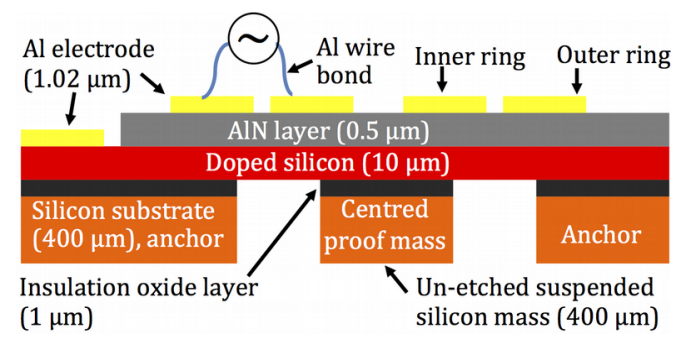

Figure 4: Cross-sectional view of stack of material.

The MEMS harvester was experimentally tested on a mechanical shaker controlled by a frequency/arbitrary waveform generator. Both sinusoidal frequency (to drive the device into resonances) and band-limited white noise excitation can be programmed into the shaker. An off-theshelf MEMS accelerometer was fixed together with the vibrating platform in order to measure the acceleration experienced by the device.

\section{RESULTS}

\section{Parametric resonance and higher orders}

Up to 8 orders of parametric resonance were recorded when the piezoelectric transducer was connected to a matched load $(\sim 70 \mathrm{k} \Omega)$. With a natural frequency $f_{n}$ of about $980 \mathrm{~Hz}$, the principal (1st order) parametric resonance was observed when the excitation frequency $f_{e x}$ was about $2 f_{n}$. However, the response frequency was always exactly half of the excitation (at around $f_{n}$ ). Figure 5 presents the FFT of the time domain voltage output of the transducer. A small peak around $f_{e x}$ represents the nonresonant directly forced response.

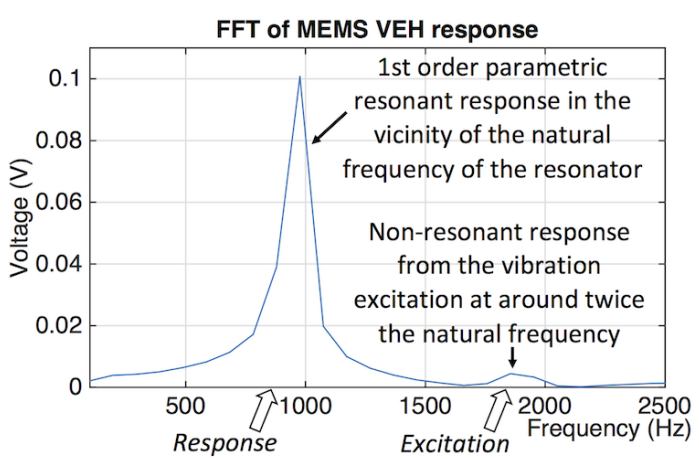

Figure 5: FFT of the voltage response when driven into the 1 st order parametric resonance (excitation frequency is twice the natural frequency: $\sim 1.9 \mathrm{kHz}$ ).
This excitation frequency to response frequency ratio is a distinct signature to identify parametric resonance and its respective order number. On the other hand, for directly excited resonance, the response frequency always matches the excitation. Figure 6 further demonstrates this distinct frequency ratio signature for the 3rd order, where the excitation frequency $f_{e x}$ is at around $640 \mathrm{~Hz}$ and the response frequency is $980 \mathrm{~Hz}$. The same trend of frequency ratio signatures for each respective order were observed up to the 8th order, where $4 f_{e x}=f_{n}$.

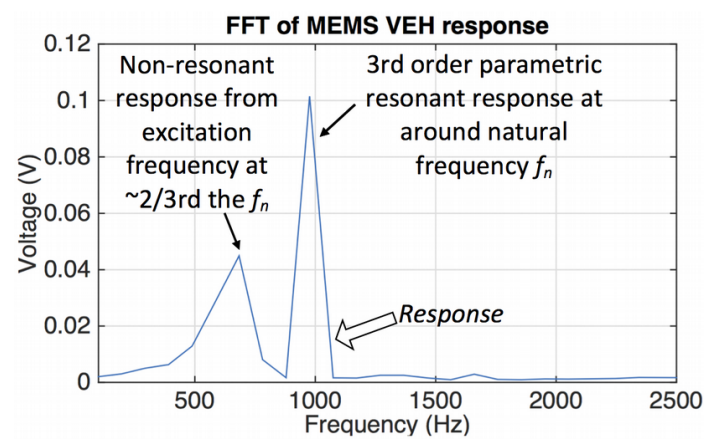

Figure 6: FFT of the voltage response when driven into the $3 r d$ order parametric resonance (excitation frequency is twice the natural frequency: $\sim 640 \mathrm{~Hz}$ ).

At even lower frequency intervals, under the electrically damped condition, no observable order was measured within the scanned acceleration range. This is partly due to the higher initiation threshold amplitude of the damped higher orders; and the fast narrowing nature of frequency bandwidth, which makes experimental measurement practically difficult.

\section{Multi-frequency operation}

The first two orders of parametric resonance and the directly excited fundamental mode were the most readily activated resonant peaks in this device. Figure 7 presents the power response per excitation frequency at $0.05 \mathrm{~g}$ of sinusoidal drive. A strong nonlinearity can be noted for the parametric resonant peaks and the resonant onset was sensitive to initial conditions. The higher order parametric resonances were not detected at this acceleration amplitude.

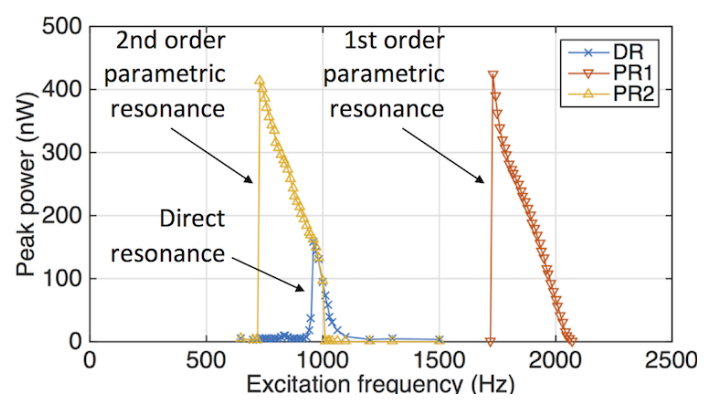

Figure 7: Frequency domain power response of the disk membrane MEMS harvester when subjected to acceleration amplitude of $0.05 \mathrm{~g}$. Power peaks for parametric resonance were both wider and higher than direct resonance. 
The response frequency of the 2 nd order coincides with that of the directly excited fundamental mode. Therefore, the identification criteria for the two overlapping resonances was the sudden rise and fall of response when scanning around the boundaries of the parametric instability region. Furthermore, these two resonant responses do not co-exist, but can switch between each other under certain boundary conditions.

The direct resonant peak itself exhibited strong Duffing nonlinearity in the form of spring softening. This, along with saturation of the parametric resonant oscillatory amplitude growth by the presence of nonlinear damping, the system can thus be modelled as a forced Mathieu Duffing oscillator with a nonlinear damping term described in equation 2 [7].

$$
\begin{gathered}
\ddot{x}+\alpha 2 \zeta \omega_{0} \dot{x}+\alpha \beta \dot{x} x^{2}+\left[\omega_{0}^{2}+\alpha \varepsilon \cos (\omega t)\right] x \\
+\alpha \mu x^{3}=\alpha F \cos (\omega t+\phi)
\end{gathered}
$$

where, $\zeta$ is the linear damping ratio, $\beta$ is the nonlinear quadratic damping coefficient, $\mu$ is the geometric Duffing nonlinearity, $\varepsilon$ is the parametric excitation, $\mathrm{F}$ is external forcing amplitude, $\phi$ is the phase difference between the external forcing and the parametric excitation, and $\alpha$ is a small parameter that can be added for use in numerical perturbation method [7].

With increasing excitation amplitude, further parametric resonances were revealed as shown in figure 8 . At $2.0 \mathrm{~g}$ of sinusoidal drive, 8 orders were recorded, albeit the higher orders exhibited relatively narrower and smaller peaks compared to the direct resonant regime.

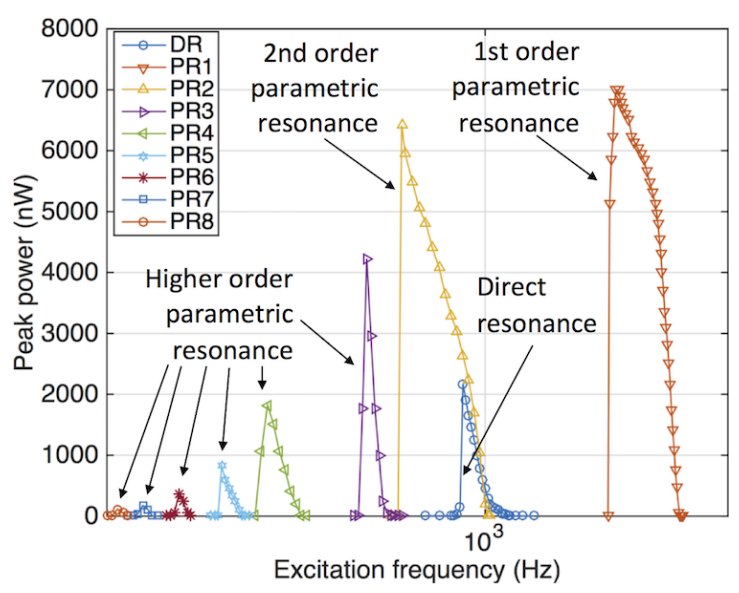

Figure 8: Frequency domain power response when subjected to sinusoidal acceleration amplitude of $2.0 \mathrm{~g}$.

Table 1 delineates the power peak and the frequency bandwidth of figure 8 . It can be seen from both the figure and the table that the first three orders can potentially yield higher power peaks than direct resonance for the same excitation acceleration amplitude. At $2.0 \mathrm{~g}$, direct resonance peaked at $2.2 \mu \mathrm{W}$, while the parametric resonant peaks attained $7.0 \mu \mathrm{W}, 6.4 \mu \mathrm{W}$ and $4.2 \mu \mathrm{W}$ for the 1 st order, 2 nd order and 3 rd order respectively.
Table 1: Comparison of direct resonance (DR) and parametric resonance $(P R)$ of the disk membrane harvester driven at $2.0 \mathrm{~g}$. HHP: half power point.

\begin{tabular}{|c|r|r|r|}
\hline \multirow{2}{*}{$\begin{array}{c}\text { Resonance } \\
\text { (peak freq. })\end{array}$} & \multirow{2}{*}{$\begin{array}{c}\text { Power } \\
\text { peak } \\
(\mu W)\end{array}$} & \multicolumn{2}{|c|}{ Freq. bandwidth $(\mathrm{Hz})$} \\
\cline { 3 - 4 } & & HPP & HPP of DR \\
\hline DR $(920 \mathrm{~Hz})$ & 2.2 & \multicolumn{2}{|c|}{$\mathbf{5 5}$} \\
\hline PR 1 $(1640 \mathrm{~Hz})$ & 7.0 & 365 & 445 \\
\hline$P R 2(730 \mathrm{~Hz})$ & 6.4 & 150 & 245 \\
\hline PR 3 $(640 \mathrm{~Hz})$ & 4.2 & 25 & 40 \\
\hline $\begin{array}{c}\text { Accumulative } \\
\text { PR 1 to PR } 8\end{array}$ & & $\mathbf{6 0 4}$ & $\mathbf{7 6 0}$ \\
\hline
\end{tabular}

In terms of frequency bandwidth, the 1 st order $(365$ $\mathrm{Hz})$ and 2nd order $(150 \mathrm{~Hz})$ demonstrated 6.6 times and 2.7 times wider half power band than the direct peak $(55$ $\mathrm{Hz}$ ), respectively. If the half power points of the direct resonant peak is taken as the reference, this bandwidth enhancement is approximately 8 times for the 1 st order and almost 4.5 times for the 2 nd order.

Furthermore, the accumulative half power bandwidth of parametric resonance is nearly 11 times wider than direct resonance, and almost 14 times wider if the half power point of the direct peak is considered as the reference. Therefore, this result illustrated the potential superior performance of parametric resonators for multifrequency operation.

\section{Broadband response}

In order to further assess the broadband response of the disk membrane parametric resonator, a typical direct resonator (fabricated with the same AIN on SOI process), using the classic cantilever topology, was employed for experimental comparison and benchmark. Figure 9 shows the MEMS cantilevers. A cantilever with $60 \%$ of its length occupied by a suspended end mass was chosen.

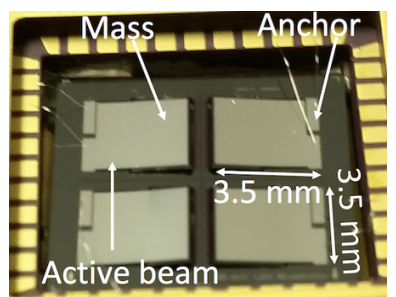

Figure 9: Photograph of the cantilever MEMS vibration energy harvesters $\left(f_{n} \sim 200 \mathrm{~Hz}\right)$, used for experimental comparison with the disk membrane.

Both the membrane resonator and the cantilever resonator were subjected to band-limited (10 $\mathrm{Hz}$ to $2 \mathrm{kHz})$ white noise vibration of varying amplitudes. Figure 10 illustrates the membrane device subjected to the noise input from the shaker, which was programmed by a computer via the arbitrary waveform generator and its intensity characterised by an accelerometer. 

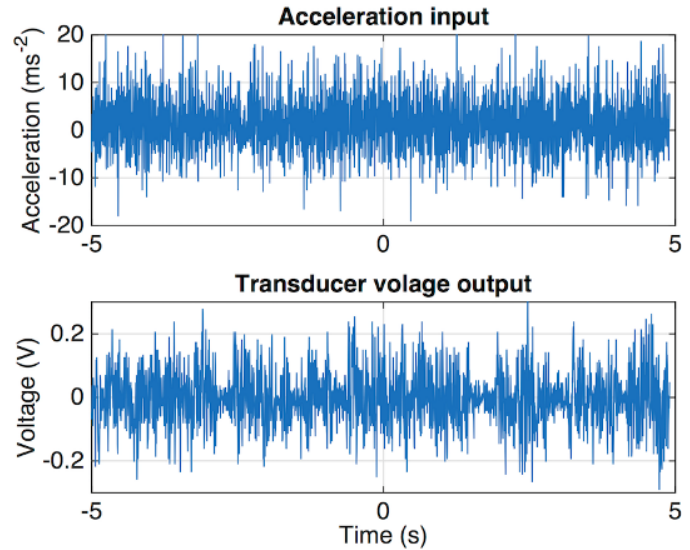

Figure 10: A typical response from the membrane device when subjected to a band-limited white noise with a noise intensity of $0.002 \mathrm{~g}^{2} / \mathrm{Hz}$. A MEMS accelerometer was employed to characterize the input noise amplitude from the programmed shaker.

Figure 11 presents the average power density response of both the membrane (parametric) resonator and the cantilever (direct) resonator for noise intensity between $0.0005 \mathrm{~g}^{2} / \mathrm{Hz}$ and $0.05 \mathrm{~g}^{2} / \mathrm{Hz}$. Due to the relatively unpredictable and random nature of the power peaks from noise excitation, average power was chosen as a more reliable parameter. The power output was averaged over intervals of 10 seconds.

The average power values were normalised against volume for the comparison due to the different sizes of the two devices. The membrane device had an effective volume of $0.154 \mathrm{~cm}^{3}$ while the cantilever occupied less than a third of the volume at $0.0049 \mathrm{~cm}^{3}$. However, the cantilever also had larger travel (a conservative estimate of $\pm 1 \mathrm{~mm}$ at large amplitudes). Therefore, if the extra travel is to be taken into account, the smaller cantilever would still require approximately similar, if not more active volume than its less compliant membrane counterpart.

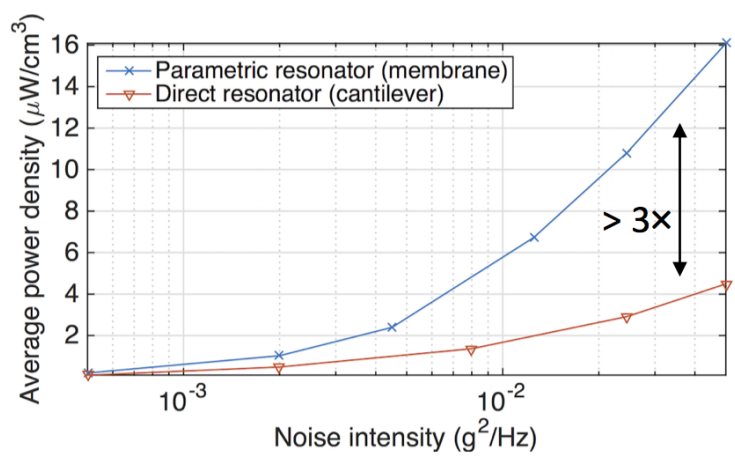

Figure 11: Measured output power response of the parametric resonator and the direct resonator for band-limited white noise excitation (10 $\mathrm{Hz}$ to $2 \mathrm{kHz}$ ).

Figure 11 illustrates up to over 3-folds enhancement in the average power output normalised to the device volume of the parametric resonator over the conventional direct resonator. The power density calculation only takes into account the device volume and not the travel (as discussed above), which thus skews the result in favour of the cantilever. Furthermore, a trend of increasing gap can also be noted for the membrane over the cantilever at larger amplitudes.

\section{CONCLUSION}

This paper presents a piezoelectric membrane resonator capable of being driven into parametric resonances for the purpose of multi-frequency vibration energy harvesting. Up to 8 orders of parametric resonance were recorded for the harvester and over an order of magnitude enhancement in operational frequency bandwidth was observed compared to the direct resonant peak of the same device.

When compared to a classic cantilever resonator fabricated using the same process, the membrane parametric resonator demonstrated over a 3-fold higher average power density when subjected to band-limited white noise excitation. This illustrates the potential of a membrane-based parametric resonator to capture a wider region of the available power spectrum.

Future work will investigate the potential to further improve the operational frequency bandwidth under vacuum conditions (parametric resonance increases both in power amplitude and frequency bandwidth at lower damping [2]), as well as exploring additional design iterations to further the proof-of-concept device reported here.

\section{ACKNOWLEDGEMENTS}

This work was supported by Innovate UK (Project reference: 131183).

\section{REFERENCES}

[1] S. Priya and D. Inman, Energy Harvesting Technologies, (Springer, 2009)

[2] Y. Jia, J. Yan, K. Soga and A.A. Seshia, "Multifrequency Operation of a MEMS Vibration Energy Harvester by Accessing Five Orders of Parametric Resonance", J. Phys. Conf. Ser., 476(1), pp. 607-611, 2013

[3] M. Faraday, Phil. Trans., "On a peculiar class of acoustical figures and on certain forms assumed by a group of particles upon vibrating elastic surfaces", pp. 299-318, 1831

[4] A. Tondl, T. Ruijgrok, F. Verhulst and R. Nabergoj, Autoparametric resonance in mechanical syst., (Cambridge, 2000)

[5] K. Turner, S. Miller, P. Hartwell, N. MacDonald, S. Strogatz and S. G. Adams, "Five parametric resoannces in a microelectromechanical system", Nature, 396, pp. 149-152, 1988

[6] A. Nayfeh and D. Mook, Nonlinear Oscillations, (Wiley-Interscience, 19749)

[7] M. Pandey, R.H. Rand and A.T. Zehnder, "Frequency locking in a forced Mathieu-van der Pol-Duffing system", Nonlinear Dyn, 54, pp. 3-12, 2008

\section{CONTACT}

*Yu Jia, Tel: +44-1244-512418; yu.jia.gb@,ieee.org 